\title{
Algebraic structures derived from BCK-algebras
}

\author{
Ivan Chajda and Jan Kühr
}




\title{
ALGEBRAIC STRUCTURES DERIVED FROM BCK-ALGEBRAS
}

\author{
IVAN CHAJDA AND JAN KÜHR \\ Received 17 January, 2006
}

\begin{abstract}
Commutative BCK-algebras can be viewed as semilattices whose sections have antitone involutions and it is known that bounded commutative BCK-algebras are equivalent to $\mathrm{MV}$-algebras. In the first part of this paper we assign to an arbitrary BCK-algebra a semilatticelike structure every section of which possesses a certain antitone mapping. The remaining part is devoted to algebras of the MV-language $\{\oplus, \neg, 0\}$ which are defined on bounded BCK-algebras in the same way as MV-algebras.
\end{abstract}

1991 Mathematics Subject Classification: 03G25, 06D35, 06F35

Keywords: BCK-algebra, MV-algebra, semilattice, antitone mapping

1.

A $B C K$-algebra is an algebra $\mathcal{A}=(A ; \rightarrow, 1)$ of type $(2,0)$ satisfying the following quasi-identities:

(I) $(x \rightarrow y) \rightarrow((y \rightarrow z) \rightarrow(x \rightarrow z))=1$,

(II) $x \rightarrow((x \rightarrow y) \rightarrow y)=1$,

(III) $x \rightarrow x=1$,

(IV) $x \rightarrow 1=1$,

(V) $(x \rightarrow y=1 \& y \rightarrow x=1) \Rightarrow x=y$.

BCK-algebras were introduced by Y. Imai and K. Iséki [5-7] and form an algebraic semantics for C. A. Meredith's logic.

The relation $\leq$ on $A$ given by

$$
x \leq y \quad \Leftrightarrow \quad x \rightarrow y=1
$$

is a partial order on $A$ with 1 as the top element, but the poset $(A ; \leq)$ has no particular properties because any poset $(P ; \leq)$ with 1 can be made a BCK-algebra $(P ; \rightarrow, 1)$ by setting $x \rightarrow y:=1$ for $x \leq y$, and $x \rightarrow y:=y$ otherwise.

By a bounded $B C K$-algebra we mean an algebra $\mathcal{A}=(A ; \rightarrow, 0,1)$, where $(A ; \rightarrow$ $, 1)$ is a BCK-algebra with the bottom element 0 .

This work was supported by the Research and Development Council of the Czech Government via project MSM6198959214. 
In every BCK-algebra $(A ; \rightarrow, 1)$, the following hold for all $x, y, z \in A$ :

$$
\begin{aligned}
& x \leq y \Rightarrow y \rightarrow z \leq x \rightarrow z, \\
& x \leq y \Rightarrow z \rightarrow x \leq z \rightarrow y, \\
& x \rightarrow(y \rightarrow z)= \rightarrow(x \rightarrow z), \quad \text { (exchange) } \\
& y \leq x \rightarrow y \\
& 1 \rightarrow x=x \\
& x \rightarrow y \leq(z \rightarrow x) \rightarrow(z \rightarrow y) \\
&((x \rightarrow y) \rightarrow y) \rightarrow y=x \rightarrow y .
\end{aligned}
$$

A commutative $B C K$-algebra is a $\mathrm{BCK}$-algebra that satisfies the identity

$$
(x \rightarrow y) \rightarrow y=(y \rightarrow x) \rightarrow x .
$$

In this case, the underlying poset is a join-semilattice in which $x \vee y=(x \rightarrow y) \rightarrow y$. Commutative BCK-algebras form a variety that is axiomatized by the identities (9), (4), (III) and (6).

We have proved in $[1,2]$ that commutative BCK-algebras (named here weak implication algebras and defined in a slightly different way) can be characterized as joinsemilattices whose sections (= principal order filters) posses antitone involutions.

Recall that by a semilattice with sectionally antitone involutions we mean a structure $\delta=\left(S ; \vee,\left({ }^{a}\right)_{a \in S}, 1\right)$, where $(S ; \vee)$ is a join-semilattice with the greatest element 1 , and for every $a \in S$, the mapping $x \mapsto x^{a}$ is an antitone involution on the section $[a, 1]=\{x \in S: a \leq x\}$. If $\mathcal{A}=(A ; \rightarrow, 1)$ is a commutative BCK-algebra, then $\delta(\mathcal{A})=\left(A ; \vee,\left({ }^{a}\right)_{a \in A}, 1\right)$, where $x \vee y=(x \rightarrow y) \rightarrow y$ and $x^{a}=x \rightarrow a$ $(x \in[a, 1])$, is a semilattice with sectionally antitone involutions in which we have $x \rightarrow y=(x \vee y)^{y}$. On the other hand, given a structure $\delta=\left(S ; \vee,\left({ }^{a}\right)_{a \in S}, 1\right)$, we define a new algebra $\mathcal{A}(\delta)=(S ; \rightarrow, 1)$ via $x \rightarrow y=(x \vee y)^{y}$. Then $\mathcal{A}(\delta)$ is a BCKalgebra if and only if it satisfies identity (4), and if this is the case, then $\mathscr{A}(\mathscr{S})$ is a commutative BCK-algebra.

Our first objective is to give a similar description for general BCK-algebras, i. e. to an arbitrary BCK-algebra we assign a semilattice-like structure the sections of which have certain antitone mappings, and also conversely, we describe the reverse passage from such structures to BCK-algebras.

Theorem 1. Let $\mathcal{A}=(A ; \rightarrow, 1)$ be a BCK-algebra. Define a binary term operation $\sqcup$ on $A$ by

$$
x \sqcup y=(x \rightarrow y) \rightarrow y,
$$

and for every $a \in A$, a unary operation ${ }^{a}$ on the section $[a, 1]=\{x \in A: a \leq x\}$ by

$$
x^{a}=x \rightarrow a .
$$

Then the structure $\mathcal{S}(\mathcal{A})=\left(A ; \sqcup,\left({ }^{a}\right)_{a \in A}, 1\right)$ satisfies the following quasi-identities:

(i) $x \sqcup x=x$, 
(ii) $(x \sqcup y=y \& y \sqcup x=x) \Rightarrow x=y$,

(iii) $x \sqcup y=(x \sqcup y) \sqcup y=x \sqcup(x \sqcup y)=y \sqcup(x \sqcup y)$,

(iv) $(x \sqcup z) \sqcup((x \sqcup y) \sqcup z)=(x \sqcup y) \sqcup z$,

(v) $x \sqcup 1=1$,

(vi) $x^{x}=1,1^{x}=x$,

(vii) $x \sqcup y=(x \sqcup y)^{y y}=\left((x \sqcup y)^{y} \sqcup y\right)^{y}$,

(viii) $(x \sqcup y)^{y} \sqcup((x \sqcup z) \sqcup(y \sqcup z))^{y \sqcup z}=((x \sqcup z) \sqcup(y \sqcup z))^{y \sqcup z}$,

(ix) $\left((x \sqcup z)^{z} \sqcup(y \sqcup z)\right)^{y \sqcup z}=\left((y \sqcup z)^{z} \sqcup(x \sqcup z)\right)^{x \sqcup z}$,

(x) $((x \sqcup y) \sqcup x)^{x}=(x \sqcup y)^{x}$.

Proof. First note that $x \sqcup y \in[y, 1]$ by (5), hence using (8) we have

$$
(x \sqcup y)^{y}=((x \rightarrow y) \rightarrow y) \rightarrow y=x \rightarrow y .
$$

Further

$$
x \leq y \quad \Leftrightarrow \quad x \rightarrow y=1 \quad \Leftrightarrow \quad x \sqcup y=y .
$$

Indeed, $x \rightarrow y=1$ implies $x \sqcup y=(x \rightarrow y) \rightarrow y=1 \rightarrow y=y$, and conversely, if $y=x \sqcup y=(x \rightarrow y) \rightarrow y$, then $1=y \rightarrow y=((x \rightarrow y) \rightarrow y) \rightarrow y=x \rightarrow y$.

Now, we can verify the properties (i) $-(\mathrm{x})$ by direct computations:

(i) $x \sqcup x=(x \rightarrow x) \rightarrow x=1 \rightarrow x=x$.

(ii) If $x \sqcup y=y$ and $y \sqcup x=x$, then $x \rightarrow y=1$ and $y \rightarrow x=1$ by (11), so that $x=y$ by axiom $(\mathrm{V})$.

(iii) We have $(x \sqcup y) \sqcup y=(((x \rightarrow y) \rightarrow y) \rightarrow y) \rightarrow y=(x \rightarrow y) \rightarrow y=x \sqcup y$, and the equalities $x \sqcup(x \sqcup y)=y \sqcup(x \sqcup y)=x \sqcup y$ follow from (11) as $x, y \leq x \sqcup y$.

(iv) Since $x \leq x \sqcup y$ implies $x \sqcup z=(x \rightarrow z) \rightarrow z \leq((x \sqcup y) \rightarrow z) \rightarrow z=(x \sqcup$ $y) \sqcup z$, we have $(x \sqcup z) \sqcup((x \sqcup y) \sqcup z)=(x \sqcup y) \sqcup z$ by (11).

(v) $x \sqcup 1=(x \rightarrow 1) \rightarrow 1=1$.

(vi) $x^{x}=x \rightarrow x=1$ and $1^{x}=1 \rightarrow x=x$.

(vii) According to (10), $(x \sqcup y)^{y y}=(x \rightarrow y) \rightarrow y=x \sqcup y$ and $\left((x \sqcup y)^{y} \sqcup y\right)^{y}=$ $((x \rightarrow y) \sqcup y)^{y}=(x \rightarrow y) \rightarrow y=x \sqcup y$.

(viii) Due to (10), we have

$$
\begin{aligned}
((x \sqcup z) \sqcup(y \sqcup z))^{y \sqcup z} & =(x \sqcup z) \rightarrow(y \sqcup z) \\
& =((x \rightarrow z) \rightarrow z) \rightarrow((y \rightarrow z) \rightarrow z) \\
& =(y \rightarrow z) \rightarrow(((x \rightarrow z) \rightarrow z) \rightarrow z) \\
& =(y \rightarrow z) \rightarrow(x \rightarrow z),
\end{aligned}
$$

whence

$$
\begin{aligned}
(x \sqcup y)^{y} \sqcup((x \sqcup z) \sqcup(y \sqcup z))^{y \sqcup z} & =(x \rightarrow y) \sqcup((y \rightarrow z) \rightarrow(x \rightarrow z)) \\
& =(y \rightarrow z) \rightarrow(x \rightarrow z) \\
& =((x \sqcup z) \sqcup(y \sqcup z))^{y \sqcup z}
\end{aligned}
$$

by (I) and (11). 
(ix) Again, in view of (10),

$$
\begin{aligned}
\left((x \sqcup z)^{z} \sqcup(y \sqcup z)\right)^{y \sqcup z} & =(x \rightarrow z) \rightarrow(y \sqcup z) \\
& =(x \rightarrow z) \rightarrow((y \rightarrow z) \rightarrow z) \\
& =(y \rightarrow z) \rightarrow((x \rightarrow z) \rightarrow z) \\
& =(y \rightarrow z) \rightarrow(x \sqcup z) \\
& =\left((y \sqcup z)^{z} \sqcup(x \sqcup z)\right)^{x \sqcup z .}
\end{aligned}
$$

(x) We have $((x \sqcup y) \sqcup x)^{x}=(((x \sqcup y) \rightarrow x) \rightarrow x) \rightarrow x=(x \sqcup y) \rightarrow x=(x \sqcup$ $y)^{x}$.

Lemma 2. Let $(A ; \sqcup)$ be a groupoid satisfying the quasi-identities (i)-(iv) of Theorem 1 . Then the binary relation defined by

$$
x \leq y \quad \Leftrightarrow \quad x \sqcup y=y
$$

is a partial order on $A$ such that, for every $x, y \in A, x \sqcup y$ is a common upper bound of $x, y$.

Proof. By (i) and (ii), $\leq$ is reflexive and antisymmetric. For transitivity, assume that $x \sqcup y=y$ and $y \sqcup z=z$. Then $x \sqcup z=(x \sqcup z) \sqcup z=(x \sqcup z) \sqcup(y \sqcup z)=$ $(x \sqcup z) \sqcup((x \sqcup y) \sqcup z)=(x \sqcup y) \sqcup z=y \sqcup z=z$ by (iii) and (iv). Thus $\leq$ is a partial order on $A$. Moreover, from (iii) we conclude that $x, y \leq x \sqcup y$.

Therefore, any BCK-algebra induces a semilattice-like structure with a join-like operation $\sqcup$. Another kind of generalizations of join-semilattices was introduced by J. Ježek and R. Quackenbush [8]:

A directoid is a groupoid $(A ; \sqcup)$ satisfying the identities
(a) $x \sqcup x=x$,
(b) $(x \sqcup y) \sqcup x=x \sqcup y$,
(c) $y \sqcup(x \sqcup y)=x \sqcup y$,
(d) $x \sqcup((x \sqcup y) \sqcup z)=(x \sqcup y) \sqcup z$.

The relation $\leq$ given by (12) is a partial order. The binary operation $\sqcup$ assigns to a pair $(x, y)$ a common upper bound of $\{x, y\}$ in such a way that $x \sqcup y=y \sqcup x=y$ provided $x \leq y$.

Observe that this is the point where directoids differ from our semilattice-like structures since in our case $x \leq y$ does not imply $y \sqcup x=y$.

Lemma 3. Let $\mathcal{A}=(A ; \rightarrow, 1)$ be a $B C K$-algebra and $\sqcup$ be the binary operation defined in Theorem 1. Then the following conditions are equivalent:

(a) $(A ; \sqcup)$ is a directoid;

(b) A is a commutative BCK-algebra;

(c) $(A ; \sqcup)$ is a join-semilattice. 
Proof. (a) $\Rightarrow$ (b). Assume that $(A ; \sqcup)$ is a directoid. Remember that $x \leq y$ iff $x \rightarrow y=1$ iff $x \sqcup y=y$. Since $(A$; $\sqcup)$ is a directoid, $x \leq y$ entails $x \sqcup y=y \sqcup x=y$, so $\mathcal{A}$ satisfies the quasi-identity

$$
x \leq y \quad \Rightarrow \quad y=(y \rightarrow x) \rightarrow x .
$$

Hence $x \leq(x \rightarrow y) \rightarrow y$ yields $(x \rightarrow y) \rightarrow y=(((x \rightarrow y) \rightarrow y) \rightarrow x) \rightarrow x$. But from $y \leq(x \rightarrow y) \rightarrow y$ it follows $(y \rightarrow x) \rightarrow x \leq(((x \rightarrow y) \rightarrow y) \rightarrow x) \rightarrow x$, and hence $(y \rightarrow x) \rightarrow x \leq(x \rightarrow y) \rightarrow y$. The converse inequality is obtained by interchanging $x$ and $y$, thus $\mathcal{A}$ is a commutative BCK-algebra.

(b) $\Rightarrow$ (c). As we already know, if $\mathcal{A}$ is a commutative BCK-algebra, then $x \sqcup y=$ $(x \rightarrow y) \rightarrow y$ is the supremum of $\{x, y\}$, hence $(A ; \sqcup)$ is a join-semilattice.

(c) $\Rightarrow$ (a). Clearly, every join-semilattice is a directoid.

Theorem 4. Let $\delta=\left(S ; \sqcup,\left({ }^{a}\right)_{a \in S}, 1\right)$ be a structure-where $\sqcup$ is a binary operation on $S$ and for each $a \in S,{ }^{a}: x \mapsto x^{a}$ is unary operation on $\{x \in S: a \sqcup x=x\}$, and 1 is a distinguished element of $S$-satisfying the quasi-identities (i)-(ix) from Theorem 1. Define a new binary operation $\rightarrow$ on $S$ by

$$
x \rightarrow y=(x \sqcup y)^{y} .
$$

Then $\mathcal{A}(8)=(S ; \rightarrow, 1)$ is a BCK-algebra.

Proof. The definition of $\rightarrow$ is correct since $y \sqcup(x \sqcup y)=x \sqcup y$ by (iii). Furthermore, we note that

$$
x \sqcup y=y \quad \Leftrightarrow \quad x \rightarrow y=1 .
$$

Indeed, if $x \sqcup y=y$, then $x \rightarrow y=(x \sqcup y)^{y}=y^{y}=1$, and conversely, $1=x \rightarrow$ $y=(x \sqcup y)^{y}$ implies $y=1^{y}=(x \sqcup y)^{y y}=x \sqcup y$.

Now, we verify the axioms of BCK-algebras:

(I) By (viii) we have

$$
\begin{aligned}
(x \rightarrow y) \sqcup((x \sqcup z) \rightarrow(y \sqcup z)) & =(x \sqcup y)^{y} \sqcup((x \sqcup z) \sqcup(y \sqcup z))^{y \sqcup z} \\
& =((x \sqcup z) \sqcup(y \sqcup z))^{y \sqcup z} \\
& =(x \sqcup z) \rightarrow(y \sqcup z),
\end{aligned}
$$

so $(x \rightarrow y) \rightarrow((x \sqcup z) \rightarrow(y \sqcup z))=1$. Further, using (vii) and (ix), we obtain

$$
\begin{aligned}
(x \rightarrow z) \rightarrow((y \rightarrow z) \rightarrow z) & =(x \rightarrow z) \rightarrow\left((y \sqcup z)^{z} \sqcup z\right)^{z} \\
& =(x \rightarrow z) \rightarrow(y \sqcup z) \\
& =\left((x \sqcup z)^{z} \sqcup(y \sqcup z)\right)^{y \sqcup z} \\
& =\left((y \sqcup z)^{z} \sqcup(x \sqcup z)\right)^{x \sqcup z} \\
& =(y \rightarrow z) \rightarrow(x \sqcup z) \\
& =(y \rightarrow z) \rightarrow((x \rightarrow z) \rightarrow z) .
\end{aligned}
$$


When we replace $x$ by $x \rightarrow z$, we get

$$
\begin{aligned}
(x \sqcup z) \rightarrow(y \sqcup z) & =((x \rightarrow z) \rightarrow z) \rightarrow((y \rightarrow z) \rightarrow z) \\
& =(y \rightarrow z) \rightarrow(((x \rightarrow z) \rightarrow z) \rightarrow z) \\
& =(y \rightarrow z) \rightarrow(x \rightarrow z)
\end{aligned}
$$

since $((x \rightarrow z) \rightarrow z) \rightarrow z=\left(\left((x \sqcup z)^{z} \sqcup z\right)^{z} \sqcup z\right)^{z}=((x \sqcup z) \sqcup z)^{z}=(x \sqcup z)^{z}=$ $x \rightarrow z$. Altogether, we have proved

$$
(x \rightarrow y) \rightarrow((y \rightarrow z) \rightarrow(x \rightarrow z))=(x \rightarrow y) \rightarrow((x \sqcup z) \rightarrow(y \sqcup z))=1 .
$$

(II) We have $(x \rightarrow y) \rightarrow y=\left((x \sqcup y)^{y} \sqcup y\right)^{y}=x \sqcup y$, hence $x \sqcup((x \rightarrow y) \rightarrow y)=$ $x \sqcup(x \sqcup y)=x \sqcup y=(x \rightarrow y) \rightarrow y$ and by (13) we obtain $x \rightarrow((x \rightarrow y) \rightarrow y)=1$.

(III) Due to (13), from $x \sqcup x=x$ it follows $x \rightarrow x=1$.

(IV) Analogously, $x \sqcup 1=1$ gives $x \rightarrow 1=1$.

(V) If $x \rightarrow y=1$ and $y \rightarrow x=1$, then $x \sqcup y=y$ and $y \sqcup x=x$ which imply $x=y$.

Remark 5. Observe that in Theorem 4 we did not employ the identity (x) of Theorem 1. Actually, this identity is useful in order to establish a one-to-one correspondence between $\mathrm{BCK}$-algebras and semilattice-like structures with sectionally antitone mappings:

Theorem 6. Let $\mathcal{A}=(A ; \rightarrow, 1)$ be a BCK-algebra and let $\delta=\left(S ; \sqcup,\left({ }^{a}\right)_{a \in S}, 1\right)$ be an algebra as in Theorem 4 satisfying (i)-(x) of Theorem 1. Then $\mathcal{A}(\mathcal{S}(\mathcal{A}))=\mathcal{A}$ and $\delta(\mathcal{A}(\mathcal{S}))=\delta$.

Proof. Let $\delta(\mathcal{A})=\left(A ; \sqcup,\left({ }^{a}\right)_{a \in A}, 1\right)$ be the structure satisfying (i)-(x) which is assigned to a given BCK-algebra $\mathcal{A}$ by Theorem 1 . Then in $\mathcal{A}(\mathcal{S}(\mathcal{A}))=(A ; \leadsto, 1)$ we have $x \leadsto y=(x \sqcup y)^{y}=((x \rightarrow y) \rightarrow y) \rightarrow y=x \rightarrow y$, so that $\mathcal{A}(\mathcal{S}(\mathcal{A}))=\mathcal{A}$.

Conversely, let $\delta=\left(S\right.$; $\left.\sqcup,\left({ }^{a}\right)_{a \in S}, 1\right)$ be a structure that satisfies (i)-(x) of Theorem $1, \mathcal{A}(\&)=(S ; \rightarrow, 1)$ its corresponding BCK-algebra (cf. Theorem 4) and $\delta(\mathcal{A}(8))=\left(S ; \mathbb{U},\left(r_{a}\right)_{a \in S}, 1\right)$. Then $x$ 巴 $y=(x \rightarrow y) \rightarrow y=\left((x \sqcup y)^{y} \sqcup y\right)^{y}=$ $x \sqcup y$, and for $x \in[a, 1], r_{a}(x)=x \rightarrow a=(x \sqcup a)^{a}=((a \sqcup x) \sqcup a)^{a}=(a \sqcup x)^{a}=x^{a}$ in view of $(\mathrm{x})$, and hence $\delta(\mathcal{A}(\delta))=\delta$.

Corollary 7. Let $\delta=\left(S ; \sqcup,\left({ }^{a}\right)_{a \in S}, 1\right)$ be an algebra satisfying (i)-(x) of Theorem 1. Then the relation defined by (12) is a partial order on $S, 1$ is the greatest element of $S$ and for every $x, y \in S, x, y \leq x \sqcup y$. Moreover, for each $a \in S, x \mapsto x^{a}$ is an antitone mapping on $[a, 1]=\{x \in S: a \leq x\}$.

The $M V$-algebras were introduced by C. C. Chang [3] as an algebraic counterpart of the Łukasiewicz many-valued propositional logic. Here we use the present simplest definition from [4]: 
An $M V$-algebra is an algebra $\mathcal{M}=(M ; \oplus, \neg, 0)$ of type $(2,1,0)$ satisfying the identities

$(\mathrm{MV} 1) x \oplus(y \oplus z)=(x \oplus y) \oplus z$

(MV2) $x \oplus y=y \oplus x$

(MV3) $x \oplus 0=x$

(MV4) $x \oplus \neg 0=\neg 0$,

(MV5) $\neg \neg x=x$,

(MV6) $\neg(\neg x \oplus y) \oplus y=\neg(\neg y \oplus x) \oplus x$.

$M V$-algebras are known to be termwise equivalent to bounded commutative BCKalgebras (see [4]):

(a) Let $\mathcal{M}=(M ; \oplus, \neg, 0)$ be an $M V$-algebra and define $x \rightarrow y=\neg x \oplus y$ and $1=\neg 0$. Then $\mathcal{A}(\mathcal{M})=(M ; \rightarrow, 0,1)$ is a bounded commutative BCK-algebra in which $x \oplus y=(x \rightarrow 0) \rightarrow y=(y \rightarrow 0) \rightarrow x$ and $\neg x=x \rightarrow 0$.

(b) Let $\mathcal{A}=(A ; \rightarrow, 0,1)$ be a bounded commutative BCK-algebra. Define $x \oplus y=$ $(x \rightarrow 0) \rightarrow y$ and $\neg x=x \rightarrow 0$. Then $\mathcal{M}(\mathcal{A})=(A ; \oplus, \neg, 0)$ is an $M V$-algebra in which $x \rightarrow y=\neg x \oplus y$.

In what follows, we are concerned with algebras in the language $\{\oplus, \neg, 0\}$ which arise from bounded (non-commutative) BCK-algebras in the same manner as $M V$ algebras.

Let $\mathcal{A}=(A ; \rightarrow, 0,1)$ be a bounded BCK-algebra and define binary operation $\oplus$ and a unary operation $\neg$ on $A$ by

$$
\begin{gathered}
x \oplus y=(x \rightarrow 0) \rightarrow y, \\
\neg x=x \rightarrow 0 .
\end{gathered}
$$

We refer to $\mathcal{M}(\mathcal{A})=(A ; \oplus, \neg, 0)$ as the induced algebra of a BCK-algebra $\mathcal{A}$. We also introduce a supplementary binary operation $\odot$ by

$$
x \odot y=(x \rightarrow(y \rightarrow 0)) \rightarrow 0 .
$$

Lemma 8. Given a bounded BCK-algebra $\mathcal{A}$, its induced algebra $\mathcal{M}(\mathcal{A})$ satisfies the following identities:

(1) $0 \oplus x=x, x \oplus 0=\neg \neg x$,

(2) $x \oplus \neg \neg y=y \oplus \neg \neg x$,

(3) $x \oplus 1=1 \oplus x=1$,

(4) $\neg \neg \neg x=\neg x$,

(5) $\neg x \oplus 0=\neg x$,

(6) $\neg \neg x \oplus y=x \oplus y$,

(7) $x \oplus \neg y=\neg y \oplus \neg \neg x$,

(8) $x \oplus(y \oplus z)=y \oplus(x \oplus z)$.

In addition, we have

(9) $x \odot 1=1 \odot x=\neg \neg x$,

(10) $x \odot y=y \odot x=\neg \neg x \odot y=\neg(\neg x \oplus \neg y)$, 
(11) $x \odot 0=0$,

(12) $\neg x \odot 1=\neg x$,

(13) $\neg(\neg x \odot \neg y)=\neg \neg(x \oplus \neg \neg y)$.

Proof. (1) $0 \oplus x=(0 \rightarrow 0) \rightarrow x=1 \rightarrow x=x$ and $x \oplus 0=(x \rightarrow 0) \rightarrow 0=\neg \neg x$.

(2) $x \oplus \neg \neg y=(x \rightarrow 0) \rightarrow((y \rightarrow 0) \rightarrow 0)=(y \rightarrow 0) \rightarrow((x \rightarrow 0) \rightarrow 0)=y \oplus$ $\neg \neg x$.

(3) $x \oplus 1=(x \rightarrow 0) \rightarrow 1=1$ and $1 \oplus x=(1 \rightarrow 0) \rightarrow x=0 \rightarrow x=1$.

(4) $\neg \neg \neg x=((x \rightarrow 0) \rightarrow 0) \rightarrow 0=x \rightarrow 0=\neg x$.

(5) This follows from (1) and (4).

(6) $\neg \neg x \oplus y=(((x \rightarrow 0) \rightarrow 0) \rightarrow 0) \rightarrow y=(x \rightarrow 0) \rightarrow y=x \oplus y$.

(7) This is a consequence of (2) and (4).

(8) $x \oplus(y \oplus z)=(x \rightarrow 0) \rightarrow((y \rightarrow 0) \rightarrow z)=(y \rightarrow 0) \rightarrow((x \rightarrow 0) \rightarrow z)=$ $y \oplus(x \oplus z)$.

(9) $x \odot 1=(x \rightarrow(1 \rightarrow 0)) \rightarrow 0=(x \rightarrow 0) \rightarrow 0=\neg \neg x$ and analogously $1 \odot x=$ $(1 \rightarrow(x \rightarrow 0)) \rightarrow 0=(x \rightarrow 0) \rightarrow 0=\neg \neg x$.

(10) We have $x \odot y=(x \rightarrow(y \rightarrow 0)) \rightarrow 0=(y \rightarrow(x \rightarrow 0)) \rightarrow 0=y \odot x$ and $\neg \neg x \odot y=(((x \rightarrow 0) \rightarrow 0) \rightarrow(y \rightarrow 0)) \rightarrow 0=(y \rightarrow(((x \rightarrow 0) \rightarrow 0) \rightarrow 0)) \rightarrow$ $0=(y \rightarrow(x \rightarrow 0)) \rightarrow 0=y \odot x$, and finally $\neg \neg x \odot y=(((x \rightarrow 0) \rightarrow 0) \rightarrow(y \rightarrow$ $0)) \rightarrow 0=\neg(\neg x \oplus \neg y)$.

(11) $x \odot 0=(x \rightarrow(0 \rightarrow 0)) \rightarrow 0=(x \rightarrow 1) \rightarrow 0=1 \rightarrow 0=0$.

(12) This is a consequence of (9) and (4).

(13) By (10) and (7) we have $\neg(\neg x \odot \neg y)=\neg(\neg y \odot \neg x)=\neg \neg(\neg \neg y \oplus \neg \neg x)=$ $\neg \neg(x \oplus \neg \neg y)$.

Lemma 9. Let $\mathcal{M}(\mathcal{A})$ be the induced algebra of a BCK-algebra $\mathcal{A}$. Then

(a) $x \leq y$ implies $x \oplus z \leq y \oplus z$ and $z \oplus x \leq z \oplus y$, and the same is true for $\odot$,

(b) $x \leq y \rightarrow \neg z$ iff $x \odot y \leq \neg z$,

where $\leq$ is the partial order of $\mathcal{A}$ defined by (1).

Proof. (a) From $x \leq y$ it follows $x \rightarrow 0 \geq y \rightarrow 0$ and hence $x \oplus z=(x \rightarrow 0) \rightarrow$ $z \leq(y \rightarrow 0) \rightarrow z=y \oplus z$. Similarly, $x \leq y$ yields $z \oplus x=(z \rightarrow 0) \rightarrow x \leq(z \rightarrow$ $0) \rightarrow y=z \oplus y$.

Now, let $x \leq y$. Then $\neg x \geq \neg y$ which implies $\neg x \oplus \neg z \geq \neg y \oplus \neg z$ and hence $x \odot z=\neg(\neg x \oplus \neg y) \leq \neg(\neg y \oplus \neg z)=y \odot z$.

(b) If $x \leq y \rightarrow \neg z$, then $x \odot y \leq(y \rightarrow \neg z) \odot y=((y \rightarrow(z \rightarrow 0)) \rightarrow(y \rightarrow 0)) \rightarrow$ $0 \leq((z \rightarrow 0) \rightarrow 0) \rightarrow 0=z \rightarrow 0=\neg z$ since $(z \rightarrow 0) \rightarrow 0 \leq(y \rightarrow(z \rightarrow 0)) \rightarrow(y \rightarrow$ $0)$. Conversely, if $x \odot y \leq \neg z$, then $y \rightarrow \neg z \geq y \rightarrow(x \odot y)=y \rightarrow((x \rightarrow(y \rightarrow$ $0)) \rightarrow 0)=(x \rightarrow(y \rightarrow 0)) \rightarrow(y \rightarrow 0)=(y \rightarrow(x \rightarrow 0)) \rightarrow(y \rightarrow 0) \geq(x \rightarrow 0) \rightarrow$ $0 \geq x$.

From now on, we will assume that a given bounded BCK-algebra $\mathcal{A}$ satisfies the law of double negation

$$
x=\neg \neg x .
$$


It should be underlined that (17) is not enough for a BCK-algebra $\mathcal{A}$ to be commutative. Indeed, for instance, the following bounded BCK-algebra obeys the law of double negation but it is not commutative as $(a \rightarrow b) \rightarrow b=b \neq 1=(b \rightarrow a) \rightarrow a$ :

\begin{tabular}{|c|cccc|}
\hline$\rightarrow$ & 0 & $a$ & $b$ & 1 \\
\hline 0 & 1 & 1 & 1 & 1 \\
$a$ & $b$ & 1 & 1 & 1 \\
$b$ & $a$ & $a$ & 1 & 1 \\
1 & 0 & $a$ & $b$ & 1 \\
\hline
\end{tabular}

Recall that a structure $(P ; \leq, \cdot, e)$ is a partially ordered monoid or briefly a pomonoid if $(P ; \cdot, e)$ is a monoid, $(P ; \leq)$ is a poset and, for all $x, y, z \in P, x \leq y$ implies $x \cdot z \leq y \cdot z$ and $z \cdot x \leq z \cdot y$.

A partially ordered commutative residuated integral monoid, a pocrim for short, is a structure $(P ; \leq, \cdot, \rightarrow, 1)$ such that $(P ; \leq, \cdot, 1)$ is a commutative po-monoid with the greatest element 1 , and for all $x, y, z \in P$,

$$
x \cdot y \leq z \quad \Leftrightarrow \quad x \leq y \rightarrow z .
$$

Lemma 10. Let $\mathcal{A}=(A ; \rightarrow, 0,1)$ be a bounded $B C K$-algebra satisfying the law of double negation (17). Then both $(A ; \leq, \oplus, 0)$ and $(A ; \leq, \odot, 1)$ are commutative po-monoids. Moreover, $(A ; \leq, \odot, \rightarrow, 1)$ is a pocrim.

Proof. First, we show that $\oplus$ is commutative:

$$
\begin{aligned}
x \oplus y & =(x \rightarrow 0) \rightarrow y \\
& =(x \rightarrow 0) \rightarrow((y \rightarrow 0) \rightarrow 0) \\
& =(y \rightarrow 0) \rightarrow((x \rightarrow 0) \rightarrow 0) \\
& =(y \rightarrow 0) \rightarrow x \\
& =y \oplus x .
\end{aligned}
$$

Further, $\oplus$ is associative:

$$
\begin{aligned}
(x \oplus y) \oplus z & =(((x \rightarrow 0) \rightarrow y) \rightarrow 0) \rightarrow z \\
& =(((x \rightarrow 0) \rightarrow y) \rightarrow 0) \rightarrow((z \rightarrow 0) \rightarrow 0) \\
& =(z \rightarrow 0) \rightarrow((((x \rightarrow 0) \rightarrow y) \rightarrow 0) \rightarrow 0) \\
& =(z \rightarrow 0) \rightarrow((x \rightarrow 0) \rightarrow y) \\
& =(x \rightarrow 0) \rightarrow((z \rightarrow 0) \rightarrow y) \\
& =x \oplus(z \oplus y) \\
& =x \oplus(y \oplus z) .
\end{aligned}
$$


Commutativity and associativity of the operation $\odot$ is a direct consequence. Now, by Lemma $8(1),(9)$ and Lemma $9($ a),$(A ; \leq, \oplus, 1)$ as well as $(A ; \leq, \odot, 1)$ is a commutative po-monoid.

In order to prove the latter statement, it suffices to note that by (b) of Lemma 9 we have $x \leq y \rightarrow \neg \neg z=y \rightarrow z$ if and only if $x \odot y \leq \neg \neg z=z$ verifying (18).

In the next theorem we characterize " $M V$-like" algebras arising from bounded BCK-algebras satisfying the law of double negation:

Theorem 11. Given a bounded BCK-algebra $\mathcal{A}=(A ; \rightarrow, 0,1)$ satisfying the law of double negation (17), the induced algebra $\mathcal{M}(\mathcal{A})=(A ; \oplus, \neg, 0)$ fulfils the identities (MV1)-(MV5) and the axioms

(A1) $\neg(\neg x \oplus y) \oplus \neg(\neg y \oplus z) \oplus \neg x \oplus z=1$,

(A2) $(\neg x \oplus y=1 \& \neg y \oplus x=1) \Rightarrow x=y$;

moreover, we have $x \rightarrow y=\neg x \oplus y$.

Conversely, let $\mathcal{M}=(M ; \oplus, \neg, 0)$ be an algebra of type $(2,1,0)$ and put $1=\neg 0$. If $\mathcal{M}$ satisfies (MV1)-(MV5), (A1) and (A2), then upon defining $x \rightarrow y=\neg x \oplus y$, the algebra $\mathcal{A}(\mathcal{M})=(M ; \rightarrow, 0,1)$ is a bounded BCK-algebra satisfying (17). In addition, $x \oplus y=(x \rightarrow 0) \rightarrow y$ and $\neg x=x \rightarrow 0$.

Proof. Let $\mathcal{A}$ be a bounded BCK-algebra with (17). Due to Lemma 8 and the identity (17) $\mathcal{M}(\mathcal{A})$ satisfies (MV1)-(MV5). Further, $\neg x \oplus y=((x \rightarrow 0) \rightarrow 0) \rightarrow$ $y=x \rightarrow y$, and hence (A1) can be written as

$$
(x \rightarrow y) \rightarrow((y \rightarrow z) \rightarrow(x \rightarrow z))=1
$$

which is just axiom (I). Similarly, (A2) can be rewritten in the form

$$
(x \rightarrow y=1 \& y \rightarrow x=1) \Rightarrow x=y
$$

which is $(\mathrm{V})$, the fifth axiom of BCK-algebras.

Conversely, let $\mathcal{M}=(M ; \oplus, \neg, 0)$ be an algebra having the required properties. By (A1) we have $(x \rightarrow y) \rightarrow((y \rightarrow z) \rightarrow(x \rightarrow z))=\neg(\neg x \oplus y) \oplus(\neg(\neg y \oplus z) \oplus$ $(\neg x \oplus z))=1$ proving (I). When we put $y=z=0$ in (A1), we obtain $x \rightarrow x=$ $x \oplus \neg x=\neg \neg x \oplus \neg x=\neg(\neg x \oplus 0) \oplus \neg(\neg 0 \oplus 0) \oplus \neg x \oplus 0=1$, i. e., $\mathcal{A}(\mathcal{M})$ fulfils (III). Now, $x \rightarrow((x \rightarrow y) \rightarrow y)=\neg x \oplus \neg(\neg x \oplus y) \oplus y=1$ which is (II). The axiom (IV) is clear: $x \rightarrow 1=\neg x \oplus 1=1$. Finally, according to (A2), $x \rightarrow y=1$ and $y \rightarrow x=1$ imply $x=y$. Altogether, $\mathcal{A}(\mathcal{M})$ is a BCK-algebra. Obviously, it is bounded and obeys (17).

In addition, $\neg x=\neg x \oplus 0=x \rightarrow 0$ and $x \oplus y=\neg(\neg x \oplus 0) \oplus y=(x \rightarrow 0) \rightarrow$ $y$.

Corollary 12. Bounded BCK-algebras satisfying the law of double negation are termwise equivalent to the class of all algebras $\mathcal{M}=(M ; \oplus, \neg, 0)$ that satisfy (MV1)(MV5), (A1) and (A2). 


\section{REFERENCES}

[1] I. Chajda, R. Halaš, and J. Kühr, "Implication in MV-algebras," Algebra Universalis, vol. 52, no. 4, pp. 377-382 (2005), 2004.

[2] I. Chajda, R. Halaš, and J. Kühr, "Distributive lattices with sectionally antitone involutions," Acta Sci. Math. (Szeged), vol. 71, no. 1-2, pp. 19-33, 2005.

[3] C. C. Chang, "Algebraic analysis of many valued logics," Trans. Amer. Math. Soc., vol. 88, pp. 467-490, 1958.

[4] R. L. O. Cignoli, I. M. L. D’Ottaviano, and D. Mundici, Algebraic foundations of many-valued reasoning, ser. Trends in Logic—Studia Logica Library. Dordrecht: Kluwer Academic Publishers, 2000, vol. 7.

[5] Y. Imai and K. Iséki, "On axiom systems of propositional calculi. XIV," Proc. Japan Acad., vol. 42, pp. 19-22, 1966.

[6] K. Iséki, "An algebra related with a propositional calculus," Proc. Japan Acad., vol. 42, pp. 26-29, 1966.

[7] K. Iséki and S. Tanaka, "An introduction to the theory of BCK-algebras," Math. Japon., vol. 23, no. 1, pp. 1-26, 1978/79.

[8] J. Ježek and R. Quackenbush, "Directoids: algebraic models of up-directed sets," Algebra Universalis, vol. 27, no. 1, pp. 49-69, 1990.

Authors' addresses

Ivan Chajda

Department of Algebra and Geometry, Faculty of Science, Palacký University Olomouc, Tomkova 40, 77900 Olomouc, Czech Republic

E-mail address: chajda@inf.upol.cz

Jan Kühr

Department of Algebra and Geometry, Faculty of Science, Palacký University Olomouc, Tomkova 40, 77900 Olomouc, Czech Republic

E-mail address: kuhr@inf.upol.cz 